\title{
TRES DOCUMENTOS SOBRE LA FUNDACION DE LA ESCUELA DE DANZA
}

Aurelio DE LOS REYeS

Como cualquier archivo histórico, el de la Secretaría de Educación Pública depara gratas y amargas sorpresas al investigador, según el caso; gratas porque encuentra lo inesperado; amargas porque no consigue los documentos que busca por haber sido dados "de baja" en una de tantas depuraciones. Aun lo inesperado llega incompleto, de inmediato saltan a la vista documentos faltantes. La sobrevivencia de algunos documentos es asombrosa y se debe a múltiples causas; con seguridad, la más importante es la distracción de quienes depuraban o porque los firmantes eran personas sobresalientes en la vida política y cultural del país, como es el caso de los documentos que encontré en ese archivo sobre el proyecto, fundación y primeros meses de vida de la Escuela de Danza de la Secretaría de Educación Pública firmados por Carlos Chávez, Xavier Villaurrutia, José Gorostiza y Rufino Tamayo, entre otros.

Como es de suponer, el expediente se encuentra incompleto; faltan, entre otros documentos, todos los firmados por Naciso Bassols, secretario de Educación Pública. De los documentos que sobreviven seleccioné los tres que doy a conocer: el primero por contener una ampliación de un memorándum de Carlos Chávez, escrito a solicitud de Bassols, y que cubre, en cierta medida, la ausencia de esos dos documentos; carece de fecha, pero el segundo, del 9 de febrero de 1932, me hace suponer que lo escribió en enero de ese año; este segundo documento corresponde a un dictamen sobre la conveniencia de fundar una escuela de danza. El documento número tres, del 15 de marzo de 1932, corresponde al proyecto del plan de trabajo y a los objetivos que debe tener la que tan sólo se llama Escuela de Danza de la Secretaría de Educación Pública.

El interés de Narciso Bassols y de Carlos Chávez de fundar una escuela de danza se desprende de la lectura del segundo documento, donde se habla del enorme interés suscitado entre los jóvenes por la creación de la Escuela de Plástica Dinámica en 1931, ya que para los cursos de 1932 se habían inscrito más de cien alumnos, a pesar de que sólo $\mathrm{Hi}^{-}$ pólito Zybine dio clases con regularidad. (Dicha Escuela de Plástica Dinámica es el inmediato antecedente de la Escuela de Danza Mexicana). 
También por la lectura de los documentos, me inclino a creer que es Bassols, ante esa demanda inesperada, el que solicita a Chávez una primer propuesta de una escuela de danza más formal; propuesta contenida en el memorándum desaparecido, pero que despertó el interés del secretario de Educación, por lo que solicitó a Chávez que lo ampliara.

Cronológicamente, entre los documentos que publico debió de haber otros; uno de los cuales contenía los comentarios de Bassols a la ampliación de la iniciativa de Chávez cuando la turna al Consejo de Bellas Artes, un cuerpo de asesores, y su solicitud a otros coreógrafos, danzarines o maestros de danza, para enviar otros proyectos para compararlos con la propuesta de Chávez, pues del dictamen de aquéllos (documento número dos), José Gorostiza, Xavier Villaurrutia y Rufino Tamayo, deduzco que hubo varias propuestas, de las que seleccionaron únicamente la de las hermanas Nellie y Gloria Campobello para, junto con la de Chávez, realizar un proyecto más elaborado. Falta tambien la solicitud de Bassols a sus asesores de precisar los objetivos de la contrapropuesta y de diseñar un programa de estudios, con el nombre de los maestros, y un presupuesto, que dio origen al documento número tres.

La publicación de los documentos seleccionados es importante porque nos acerca con precisión a la creación de la Escuela de Danza que se documenta en las historias de la danza mexicana sólo en una entrevista a Ana Mérida de Antonio Luna Arroyo incluida en su libro Ana Mérida en la historia de la danza mexicana moderna, editado en 1959 por Ediciones México-Abril. Asimismo es importante porque nos permite apreciar la visión que tuvieron los responsables de la creación de la Escuela de Danza para el surgimiento de una danza mexicana original.

En el dictamen de los asesores (documento número dos) sólo sobrevivió la propuesta de Carlos Chávez de nombrar director de la Escuela a un pintor que hubiese mostrado en su obra haber comprendido y asimilado de la expresión artística de los indígenas, pues Chávez proponía que la danza mexicana debía nacer a partir de los bailes de los indígenas mediante un proceso de superación y de recreación eminentemente pragmático (no habría teoría, ni aprendizaje, ni alumnos los dos primeros años); debía ser pintor porque únicamente los pintores jóvenes habían "demostrado su sensibilidad plástica y su capacidad para comprender los problemas artísticos, en su obra misma de pintores"; y porque todavía no había escenógrafo o coreógrafo capaz de orientar o encauzar las danzas indígenas a expresiones coreográficas diferentes mediante un "proceso constante de superación a si mismos sin imponerles ninguna in- 
fluencia externa"; Chávez pretendía traer a la ciudad de México danzantes indígenas y mestizos de "las regiones en donde se encuentran las expresiones más características y de mayor valor estético"; debían ser también músicos "para que de esta manera rindan completo el fenómeno musical y coreográfico". Los asesores toman la idea de Chávez aunque no los argumentos, pues dicen que debe ser un pintor "tanto porque los bailarines profesionales no tienen facultades de organización administrativa y técnica, como por el hecho de que tratarían de imponer sus particulares métodos profesionales". No extraña, pues, el nombramiento de Carlos Mérida como primer director de la nueva Escuela, porque desde su primera exposición en México, en noviembre de 1920, mostró haber asimilado motivos indígenas y técnicas y conceptos de las vanguardias europeas, principalmente de los cubistas y futuristas.

También según el dictamen de los asesores, la propuesta de las hermanas Campobello consistía en que las Misiones Culturales debian investigar las danzas regionales más importantes del país, para nutrir la docencia coreográfica a fin de crear una "Escuela Nacional, al modo de la Escuela Rusa o de la Escuela Francesa". La propuesta de Chávez excluía la docencia.

En el proyecto de los asesores sobresale su concepto del indígena, al que se consideraban incapaz de la docencia: "su única expresión es, precisamente, la danza que no pueden transmitir, porque no pueden analizar ni explicar (Danzan con los pies. Explicarían la danza con los pies)." Otro aspecto importante del documento corresponde a la necesidad, que admiten, de una escuela de danza para proporcionar modo de vivir a quienes tienen esa inquietud; esto es, ven en el bailarín a un profesional, no soalmente a un buscador de "adornos personales innecesarios". El nuevo profesionista sería dueño "de la mejor y más amplia técnica del baile", de un trabajo "de creación en el sentido de lo mexicano".

El tercer documento parte de las conclusiones del dictamen eriquecidas con el proyecto del primer programa de estudios para la nueva carrera de bailarín profesional; se cursaría en tres años; el primero se dedi. caría "preferentemente al desarrollo armónico del cuerpo, y el último a la experimentación de los ritmos plásticos mexicanos, debidamente estudiados y depurados, y a la creación personal”, No consideraba crear un cuerpo de bailarines profesionales, un ballet, adscrito al Departamento de Bellas Artes, seguramente porque era un problema a futuro, El siguiente documento en el expediente, en orden cronológico, corresponde al acuerdo número treinta del Consejo de Bellas Artes, firmado por su 
presidente, Luis Padilla Nervo, fechado el 14 de abril de 1932, en el que adjunta el dictamen y el proyecto de programa de estudios a Bassols, para que éste resuelva lo que juzgue conveniente.

La decisión debió de ser favorable, porque el 22 de abril de 1932 José Gorostiza informa al subsecretario de los tres posibles locales para la nueva escuela: la Sala de las Discusiones Libres en el ex-convento de San Pedro y San Pablo, uno en Bucareli 85 y otro en unos salones de la propia Secretaría; a cualquiera de los tres habría que hacerle adaptaciones. Se optó por el último. El folleto promocional, ilustrado por Carlos Mérida, transcribe el acuerdo número treinta ya mencionado, con los propósitos de la escuela, el reglamento y el programa de estudios y los nombres de los funcionarios y de los primeros maestros : Director, Carlos Mérida; Ayudante, Nellie Campobello; Técnica de Baile, Hipólito Zybine; Baile mexicano, Gloria Campobello; Plástica escénica, Agustín Lazo y Carlos Orozco Romero; Música, Francisco Domínguez; Bailes populares y extranjeros, Rafael Díaz; Baile teatral, Evelyn Eastin; acompañamiento, Angela Tercero, Consuelo Cuevas Ney y Jesús Durán Ruiz. Las días 15 y 16 de noviembre de 1932 se llevó a cabo el primer reconocimiento teatral cuatrimestral de los estudiantes, de lo cual deduzco que la fundación de la escuela se llevó a cabo entre julio y agosto. Se dice, en el programa, que la Escuela se creó para poner "al alcance de nuestra juventud enseñanzas y útiles indispensables para contribuir, con trabajo seriamente experimental de expresión por medio del baile y a preparar las bases de una coreografía mexicana moderna".

Según ese programa, hubo cambios en la planta de profesores: Linda Costa, K. Adamchewski e Hipólito Zybine enseñaban técnicas de baile. Especialidades: Evelyn Eastin, acrobático y Tap; Rafael Díaz y Carmen Delgado, bailes regionales españoles; Gloria Campobello y Francisco Domínguez, ritmos mexicanos; Carlos Orozco Romero, plástica escénica.

$Y$ hasta aquí los documentos sobrevivientes. Como con seguridad en el futuro el archivo será depurado, y para que no se pierdan estas precisiones en las historias de la danza, contra mi costumbre, publico estos documentos, fundamentales para aclarar la fundación de la Escuela de Danza de la Secretaría de Educación Pública. 
Sr. licenciado don Narciso Bassols, Secretario de Educación Pública.

Presente.

Me permito enviar a usted en seguida mis puntos de vista acerca de la Escuela Mexicana de Danza, que extiende mi criterio expuesto en memorándum anterior, en el sentido de que es preciso crear la danza mexicana como síntesis de las danzas indígenas y mestizas de todo el país, trayendo danzantes de diversas regiones; y lo rectifica en el sentido de que ahora creo que no es conveniente abordar por lo pronto las actividades docentes relativas, en tanto no haya una base concreta de que partir.

\section{La danza mexicana}

Las danzas mexicanas se encuentran dispersas entre las diferentes tribus indígenas y los núcleos mestizos del país siendo en cada caso una expresión particular dentro de las costumbres o ritos mágicos o religiosos de tales tribus o núcleos. Debe hacerse una reunión de todas esas expresiones diversas que viven en cada región, para lograr una expresión total.

Dicha reunión debe ser material, es decir traer danzantes de cada región a la escuela de danza mexicana; y mediante un proceso de elaboración especial que tienda primero a concretar y confirmar las características especiales de la coreografía mexicana y después a establecer un desarrollo constante y progresivo de dichas características. Es decir, la escuela de danza mexicana debe hacer que los indígenas con que trabaje, confirmen y posteriormente desarrollen estéticamente sus expresiones coreográficas propias, como resultado del ejercicio constante de la danza.

Aceptados estos puntos, lo que la Escuela de Danza de la Secretaría de Educación debe hacer, a mi juicio, es aprovechar su presupuesto de profesores para nombrar exclusivamente indígenas y designar como encauzador, director de los trabajos, a un individuo que no sea bailarín, sino que tenga la capacidad estética y las orientaciones generales en materia de arte, necesarias para ir concretando las expresiones coreográficas de los indios y para sujetar a éstos a un proceso constante de superación a sí mismos sin imponerles ningu. na influencia externa. Un orientador o encauzador de esta naturaleza, creo que puede ser elegido entre cualquiera de los pintores mexicanos jóvenes, ya que éstos han demostrado su sensibilidad plástica y su capacidad para comprender los problemas artísticos, en su obra misma de pintores.

Este plan supone la negación de todos los esfuerzos hechos por los bailarines profesionales o semiprofesionales de la ciudad de México; a los cuales evidentemente les ha faltado la orientación conveniente.

$\mathrm{Al}$ aceptar este punto de vista, asumo que no es posible establecer actividades docentes en materia de danza mientras no exista la danza mexicana como un valor artístico objetivo. De manera que no podrá haber inscripciones ni aceptación de alumnos o satélites de ninguna clase. Primero debe existir la danza mexicana como una realidad, para que a la vuelta de uno o dos años la danza mexicana sea susceptible de ser enseñada a la juventud escolar. 
Durante uno o dos años, las actividades de la escuela de danza serán, pues, eminentemente prácticas y consistirán en realizar bailables cortos o de grandes dimensiones, que al mismo tiempo que estén fortificando las características del ballet mexicano, estén sirviendo de recreación en escuelas, parques y teatros.

Al seleccionar los danzantes indígenas y mestizos, será preciso naturalmente dar preferencia a las regiones en donde se encuentren las expresiones más características y de mayor valor estético. Asimismo deberá procurarse que los danzantes elegidos sean también músicos para que de esta manera rindan completo el fenómeno musical y coreográfico. En las tres plazas de acompañantes al piano, podrán ser nombrados tres músicos elegidos en el campo o en la ciudad, pero que en todo caso sean capaces de absorber y asimilar la música mexicana y que actuarán en los actos de danza como conjunto musical acompañante, del que la Escuela de Danza dispondrá en permanencia.

Aceptado este plan y este punto de vista, recae la responsabilidad completa de los trabajos en la persona que los encauce y ésta no necesita necesariamente estar ligada a ninguna organización escolar.

CARLOS CHAVEZ (rúbrica)

\section{(Copia) MPC.}

En vista de que el Consejo de Bellas Artes determinó que la Escuela de Baile debería depender directamente del Departamento de Bellas Artes, pronunciándose así tácitamente en favor de la idea de la existencia de una Escuela de Baile, los suscritos piensan que el presente dictamen debe contraerse exclusivamente a las proposiciones presentadas por la Srita. Nellie Campobello y por el Sr. Carlos Chávez Ramírez tendientes a que el presupuesto de dicha Escuela se destine a otros propósitos.

El presente dictamen no implica, por consiguiente, un desarrollo nuevo de la cuestión, sino una cuestión previa que debemos analizar.

\section{PROPOSICION CAMPOBELLO}

El presupuesto de la Escuela debe destinarse a integrar un grupo de bailarines profesionales, entre los que figurarían las Sritas. Campobello, así como algunos otros profesores y alumnos distinguidos, que se dedicaría a crear, aprovechando los elementos coreográficos combinados de las diferentes danzas regionales del país, una Escuela Mexicana de ballet.

\section{CRITICA}

1.--En nuestro concepto, el baile, considerado como una Escuela Nacional, al modo de la Escuela Rusa o de la Escuela Francesa, es el resultado de una 
tradición que no es posible improvisar en un laboratorio sin riesgo de caer en un artificio semejante al que entre nosotros se produjo en el arte decorativo por la aplicación de un método convencional que quiso constituirse a sí mismo en una síntesis de los elementos de la antigua decoración indígena mexicana.

II.-Este grupo de profesionales estaría incapacitado para realizar los trabajos de investigación en que debería fundarse la obra de creación que proponen las Sritas. Campobello, ya que, de una manera general, necesitaría radicar en esta capital para ensayar y presentar los bailes que fueran inventando.

En cambio, los profesores misioneros están en mejores condiciones para recoger, en las fuentes mismas, toda la documentación que fuere preciso, la cual, sujeta a un trabajo de depuración por parte de un cuerpo erudito (que bien podría ser las Academias de Investigación del Conservatorio Nacional de Música), proporcionaría, debidamente clasificada y ordenada, la posibilidad de que el grupo de profesionales reconstruyera los bailes regionales e intentara trabajos tendientes a la producción de un genuino ballet mexicano.

El cuerpo de baile se limitaría entonces a estar al servicio de las academias de investigación. Pero a esta posibilidad se pueden presentar tres objeciones:

1/a.-No hay en México diez profesionales suficientemente aptos para coin. cidir en una labor hasta cierto punto unánime como debe ser toda obra de creación.

2/a.-Que, aun cuando los hubiera, no habría manera de multiplicar el grupo o prolongar su vida a causa de la ausencia de trabajos docentes, de modo que cada año nos encontraríamos con un grupo de diferente formación que necesitaría empezar nuevamente, desde sus prin. cipios, los trabajos de creación experimentados o abandonados por sus antecesores.

3/a.-Las Misiones Culturales, a cuyo cargo quedaría la recolección de los documentos folklóricos necésarios, no están preparadas para realizar dicho trabajo con la debida eficacia.

Los suscritos comprenden, sin embargo, que estos obstáculos pueden desaparecer con más o menos dificultades en el curso de algunos meses, pero quedaría siempre por resolver el problema de la duración y continuidad de la obra, y la única solución posible es la existencia de la Escuela de Baile; en otros términos, la Escuela de Baile no sólo no excluye los trabajos de investigación que proponen las Sritas. Campobello sino que los favorece en tal forma que podríamos asegurar que, sin el ambiente necesario de una escuela, fracasaría todo intento improvisado de creación.

Por otra parte, es necesario señalar, así sea incidentalmente, que la Secretaría de Educación, al renunciar a la idea de establecer una Escuela de Baile, para destinar su presupuesto a la formación de un grupo de bailarines profesionales, dejaría sin satisfacer la legítima aspiración de muchas personas que desean proporcionarse, por medio de esta actividad, no un adorno personal innecesario sino la subsistencia misma. 
(El año pasado, a pesar de que sólo el profesor Hipólito Zybine dio regularmente sus clases, porque no existió propiamente la Escuela de Baile, hubo una inscripción de más de cien alumnos, de los cuales se examinaron cuarenta).

\section{PROPOSICION CHAVEZ}

"Aprovechar el presupuesto asignado a la Escuela de Danza para nombrar, exclusivamente, indígenas de cada región de la República que realicen bailables que sirvan de recreación en escuelss, parques y teatros.

No deben existir actividades docentes en materia de danza, mientras no exista la danza mexicana, creada como una síntesis de las danzas indígenas y mestizas de todo el país."

\section{CRITICA}

I. Antes de pensar en la necesidad de aprovechar un presupuesto, debe plantearse un problema más complejo. ¿La Secretaría de Educación tiene o no interés en satisfacer la necesidad estética de las personas que desean ex. presarse por medio del arte de la danza? (Durante el año pasado, acudieron a inscribirse en número mayor de ciento a la incipiente Escuela de Plástica Dinámica). No se trata, pues, de aprovechar un presupuesto sino de satisfacer una necesidad de educación estética.

Traer danzantes de las diversas regiones de la República no soluciona en modo alguno el problema de la satisfacción de la necesidad de aprender a danzar. Fruto de una tradición muy larga, los danzantes indígenas son excelentes bailarines, a veces, pero son imposibles profesores. Su única expresión es, precisamente, la danza que no pueden trasmitir, porque no pueden analizar ni explicar. (Danzan con los pies. Explicarían la danza con los pies).

II. Realizar bailables que sirvan de recreación en escuelas, parques y teatros no es, sino muy lejanamente, una labor educacional. A la Secretaría le toca enfrentarse con los problemas y no torearlos, ni menos aún encubrirlos con artificiales actos de recreación. Aprovechar un conjunto de danzantes autóctonos dispares en espectáculos públicos, sin más aplicación inmediata determinada, no lleva a ningún fin educativo ni de creaciốn colectiva ni personal, aparte de que la labor de recreación pública en parques y teatros corresponde al Departamento Central, y ya la ejercitan las empresas teatrales comerciales.

III. Al negar las actividades docentes en materia de danza, el Sr. Chávez Ramírez no piensa que antes de lanzar a los estudiantes de cualquiera actividad artística al ejercicio libre de ésta, deben hallarse en posesión de una suma de conocimientos que podríamos llamar científicos que les faciliten el manejo de los útiles con que el artista trabaja: colores, sonidos, palabras, etc.

El Sr. Chávez Ramírez confunde, pues, la técnica con el arte mismo. Al negar la posibilidad de trasmitir estos conocimientos técnicos, niega la esencia misma de la educación. El educador puede trasmitir una y cien maneras de manejar los materiales estéticos. No puede trasmitir el genio creador, pero 
puede facilitar, así sea por reacción, explicando las diversas técnicas, la expresión del genio creador.

Expone el Sr. Chávez Ramírez que la danza mexicana se encuentra dispersa entre las diferentes tribus indígenas y núcleos mestizos del país siendo en cada caso una expresión particular de dichas tribus y núcleos. "Debe -añade- hacerse una reunión de todas estas experiencias para lograr una expresión de todas estas experiencias para lograr una expresión total".

Sería curioso pensar en este razonamiento para la creación de la canción o de la comida mexicana. Sería el sistema del puchero o de la olla podrida. Para crear la comida mexicana se necesita echar en la olla el mole poblano, el pozole, los huevos rancheros, etc., etc. El resultado será la comida mexicana.

La creación de la danza mexicana no es un acto voluntario (en caso de ser posible). No hay duda que el pueblo español sabe bailar. No hay duda que el baile español existe. Pero existe no como una suma de la jota, de la sardana, del fado y del zorcico, sino por las expresiones particulares y a un tiempo universales humanas de cada uno de estos bailes.

\section{CONCLUSIONES}

En vista de las razones expuestas, los suscritos se permiten proponer las siguientes conclusiones:

I. Que no se acepten las proposiciones arriba discutidas, pero que la cuestión previa: ¿debe o no existir la Escuela de Baile?, se resuelva por la existencia de la Escuela.

II. Que esta Escuela deberá funcionar conforme a los siguientes lineamientos:

a) Dependerá directamente del Departamento de Bellas Artes por las razones expuestas en anterior dictamen por los Consejeros señores Ordóñez y Tamayo, a saber: Que la danza, bastándose a sí misma, como todo arte independiente, estaría sometida a influencias extrañas si se le asimilara a la música o a las artes plásticas.

b) Que el objeto inmediato de la Escuela sea, dentro de lo posible, la formación de bailarines profesionales, dueños de la mejor y más amplia técnica del baile, pero orientados siempre hacia los trabajos de creación en el sentido de lo mexicano.

c) Que el último año de la carrera se dedique, como coronación del aprendizaje de la coreografía, a reconstruir o recrear nuestros bailes utilizando al efecto el material que proporcionen las Misiones Culturales, clasificado y organizado debidamente por las Academias de Investigación.

En el último año, se necesitará de una colaboración efectiva por parte de nuestros pintores, escenógrafos, músicos y literatos.

d) Lo anterior no quiere decir que deban desaparecer las manifestaciones públicas de los primeros años del aprendizaje, pues los suscritos creen 
que, por lo menos, los profesionales de la Escuela deberán dar exhibiciones periódicas como una demostración, muy eficaz desde el punto de vista objetivo, de las enseñanzas que impartieron a sus alumnos.

e) Que el Director de la Escuela deberá ser, preferentemente, un pintor, tanto porque los bailarines profesionales no tienen facultades de organización administrativa y técnica, como por el hecho de que tratarían de imponer sus particulares métodos profesionales.

III. Que, basándose en los puntos indicados en la conclusión anterior, la Comisión nombrada en el seno del Consejo formule el plan de estudios de la Escuela y proponga el personal que deberá hacerse cargo de ella, dentro de un plazo no mayor de 45 días.

México, D. F., 9 de febrero de 1932

José Gorostiza

Xavier Villaurrutia

\section{Rufino Tamayo}

\section{LA ESCUELA DE DANZA DE LA SECRETARIA DE EDUCACION $P U B L I C A$}

\section{Tendencia y Programa}

En la obra de Educación Artística que se propone desarrollar la Secretaría de Educación Pública, la creación de una Escuela de Danza, independiente de las Escuelas de Pintura, Escultura y Música, se hacía indispensable, porque si bien es verdad que la danza requiere, para cobrar toda su intensidad, el auxilio de las artes plásticas y musicales, no es menos cierto que tiene fuerza propia e independencia entre las bellas artes. Su enseñanza tiene que ser, pues, un fin y no un complemento de actividades plásticas o musicales.

El Consejo de Bellas Artes determinó que la Escuela deberá funcionar conforme a los lineamientos siguientes:

a) Dependiendo directamente del Departamento de Bellas Artes.

b) Que su objeto inmediato sea, dentro de lo posible, la formación de bailarines profesionales, dueños de la mejor y más amplia técnica del baile, pero orientados siempre hacia los trabajos de creación en el sentido de lo mexicano.

c) Que el último año de la carrera se dedique, como coronación del aprendizaje de la coreografía, a reconstruir o recrear nuestros bailes utilizan- 
do al efecto el material que proporcionen las Misiones Culturales, clasificado y organizado debidamente por las Academias de Investigación, procurando al mismo tiempo, una colaboración efectiva por parte de pintores, escenógrafos, músicos y literatos.

d) Lo anterior no quiere decir que deban desaparecer las manifestaciones públicas de los primeros años del aprendizaje, pues, por lo menos los profesionales de la Escuela, deberán dar exhibiciones periódicas como una demostración muy eficaz, desde el punto de vista objetivo, de las enseñanzas que impartieron a sus alumnos.

e) Que el Director de la Escuela deberá ser, preferentemente, un pintor, tanto porque los bailarines profesionales no tienen facultades de organización administrativa y técnica, como por el hecho de que tratarían de imponer sus particulares métodos profesionales.

Basándonos en los puntos señalados, formulamos a continuación el Plan de Estudios de la Escuela y proponemos el personal que, a nuestro juicio, está capacitado para desarollar las ideas de enseñanza artística y profesional en la Escuela en cuestión.

Queriendo proceder de un modo lógico y pensando en la imposibilidad de resolver súbitamente el complejo problema de la coreografía mexicana moderna, el Programa sigue un método que va de lo simple a lo compuesto, en un proceso de marcha racional. De los tres años en que estará dividida la carrera, el primer año se dedicará, preferentemente, al desarrollo armónico del cuerpo, y el último a la experimentación de los ritmos plásticos mexica-. nos, debidamente estudiados y depurados, y a la creación personal. Las clases de bailes mexicanos y las de especialidades se impartirán en todos los años.

\section{PRIMER AÑO}

Baile Clásico. (Ejercicios. Movimientos expresivos naturales).

Bailes Mexicanos. (Iniciación).

Especialidad.

Plástica.

\section{SEGUNDO AÑO}

Baile Clásico. (Ejercicios, solos y conjuntos),

Bailes Mexicanos.

Especialidad.

Plástica.

TERCER AÑO

Especialidad.

Creación Personal.

Elaboración de bailes mexicanos. Ballet. 
Las Especialidades que se impartan en la Escuela de Danza serán las siguientes: Baile Griego, (Duncanismo); Bailes Populares extranjeros, (Ruso, Español, Portugués), etc.; Baile Teatral Moderno, (Tap, Acrobático, etc.).

Los alumnos estarán obligados a cursar, durante los dos primeros años, dos especialidades.

El curso de Plástica, obligatorio en los dos primeros años, comprenderá: la enseñanza del dibujo en su relación con el arte de la Danza. Maquillaje. Plástica escénica. Proyectos de Decoración. Proyectos de trajes, etc.

\section{PERSONAL:}

El presupuesto actual de la Secretaría de Educación Pública señala para la Escuela de Danza el personal siguiente:

1 Director

1 Profesor de Danza

10 Profesores

3 Acompañantes

Para lograr el funcionamiento pleno de la Escuela de Danza conforme al Programa que presentan, los suscritos piensan en el personal siguiente:

1 Director.

1 Profesor de Danza (Subdirector o Revisor).

7 Profesores de Danza.

2 Profesores de Plástica.

1 Músico.

3 Acompañantes.

Y proponen, para desempeñar cada una de estas funciones, a las siguientes personas que han demostrado, algunas, su capacidad para llenar su cometido, puesto que trabajaron ya, el año próximo pasado, en las actividades de danza de la Secretaría, o que son conocidas, otras, como especialistas en las materias que tendrán a su cargo.

1 Director.

1 Profesor de Danza (Revisor de ias actividades técnicas de la Escuela, colaborador, con el músico y los pintores, en la elaboración de los experinentos del tercer año).

1 Profesor de Bailes Mexicanos (1/o, y 2/o. años).

Carlos Mérida.

1 Profesor de Baile Clásico (1/o. y 2/o. años)

1 Profesor de Baile Griego.

1 Profesor de Bailes Populares extranjeros.

1. Profesor de Baile Teatral.

2 Profesores de Plástica.

Nelie Campobello.

Gloria Campobello.

Hipólito Zybine.

Srita. Sneath.

Sr. Héctor Díaz.

Sr. Pedro Rubín

Agustín Lazo y

Carlos Orozco. 
1 Músico.

3 Acompañantes.
Francisco Domínguez - Angel Salas.

(los ya nombrados).

Tanto la Srita. Gloria Campobello como el señor Hipólito Zybine tendrían, al mismo tiempo que dos nombramientos, la doble obligación de impartir a los grupos de primero y segundo años los conocimientos de Baile Mexicano y Baile Clásico respectivamente.

Encontrándonos una vez más ante una situación de hecho, puesto que la Escuela de Danza funcionó, aunque de una manera informal, el año pasado, durante el presente año se impartirán solamente los dos primeros años de la carrera, y se preparará, con tiempo y cuidado, el trabajo de laboratorio que servirá el próximo como material para los experimentos de creación del ballet mexicano que son materia del tercer año de estudios.

Un trabajo de realción entre las Misiones Culturales y el Departamento de Bellas Artes en lo que toca al material folklórico recogido por los maestros misioneros se hace indispensable.

Los suscritos piensan que, para el año próximo sería interesante contar con una cátedra más de Especialidad (Baile Oriental), que la limitación presupuestal del presente año hace imposible pero que, acaso, la ampliación de la partida correspondiente haga posible.

Piensan, también, que la Escuela de Danza deberá empezar a funcionar desde luego aún sin esperar a que los profesores de cada materia, de común acuerdo con el Director de la Escuela, presenten al Consejo de Bellas Artes, para su aprobación, los programas detallados de su especialidad.

México, D. F., 15 de marzo de 1932

Xavier Villaurrutia

José Gorostiza 